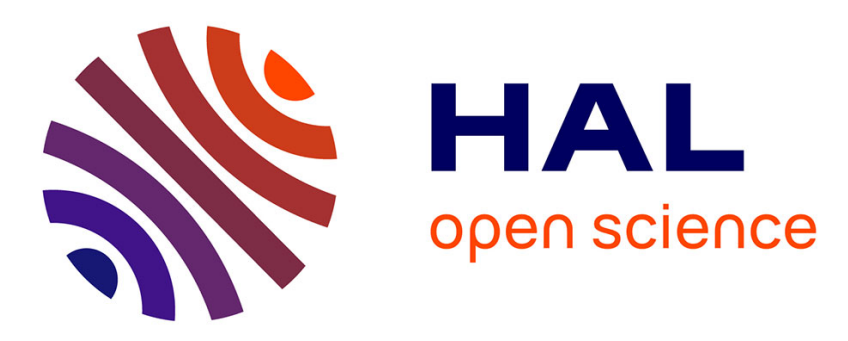

\title{
Growth Dynamics of Gallium Nanodroplets Driven by Thermally Activated Surface Diffusion
}

\author{
Zhaslan Baraissov, Federico Panciera, Laurent Travers, Jean-Christophe
} Harmand, Utkur Mirsaidov

\section{> To cite this version:}

Zhaslan Baraissov, Federico Panciera, Laurent Travers, Jean-Christophe Harmand, Utkur Mirsaidov. Growth Dynamics of Gallium Nanodroplets Driven by Thermally Activated Surface Diffusion. Journal of Physical Chemistry Letters, 2019, 10 (17), pp.5082-5089. 10.1021/acs.jpclett.9b01563 . hal02401188

\section{HAL Id: hal-02401188 \\ https://hal.science/hal-02401188}

Submitted on 9 Dec 2019

HAL is a multi-disciplinary open access archive for the deposit and dissemination of scientific research documents, whether they are published or not. The documents may come from teaching and research institutions in France or abroad, or from public or private research centers.
L'archive ouverte pluridisciplinaire $\mathbf{H A L}$, est destinée au dépôt et à la diffusion de documents scientifiques de niveau recherche, publiés ou non, émanant des établissements d'enseignement et de recherche français ou étrangers, des laboratoires publics ou privés. 


\title{
Growth Dynamics of Gallium Nanodroplets Driven by Thermally Activated Surface Diffusion
}

Zhaslan Baraissov ${ }^{1,2}$, Federico Panciera ${ }^{1,2,3 *}$, Laurent Travers $^{3}$, Jean-Christophe Harmand ${ }^{3}$, and Utkur Mirsaidov $1,2,4,5 *$

1. Department of Physics, National University of Singapore, Singapore 117551, Singapore

2. Centre for BioImaging Sciences, Department of Biological Sciences, National University of Singapore, Singapore 117557, Singapore

3. Centre for Nanoscience and Nanotechnology, CNRS, Université Paris-Sud, Université Paris-Saclay, Avenue de la Vauve, 91120 Palaiseau, France.

4. Centre for Advanced 2D Materials and Graphene Research Centre, National University of Singapore, Singapore 117546, Singapore

5. Department of Materials Science and Engineering, National University of Singapore, Singapore 117575, Singapore

*Correspondence: UM: mirsaidov@nus.edu.sg, FP: federico.panciera@c2n.upsaclay.fr

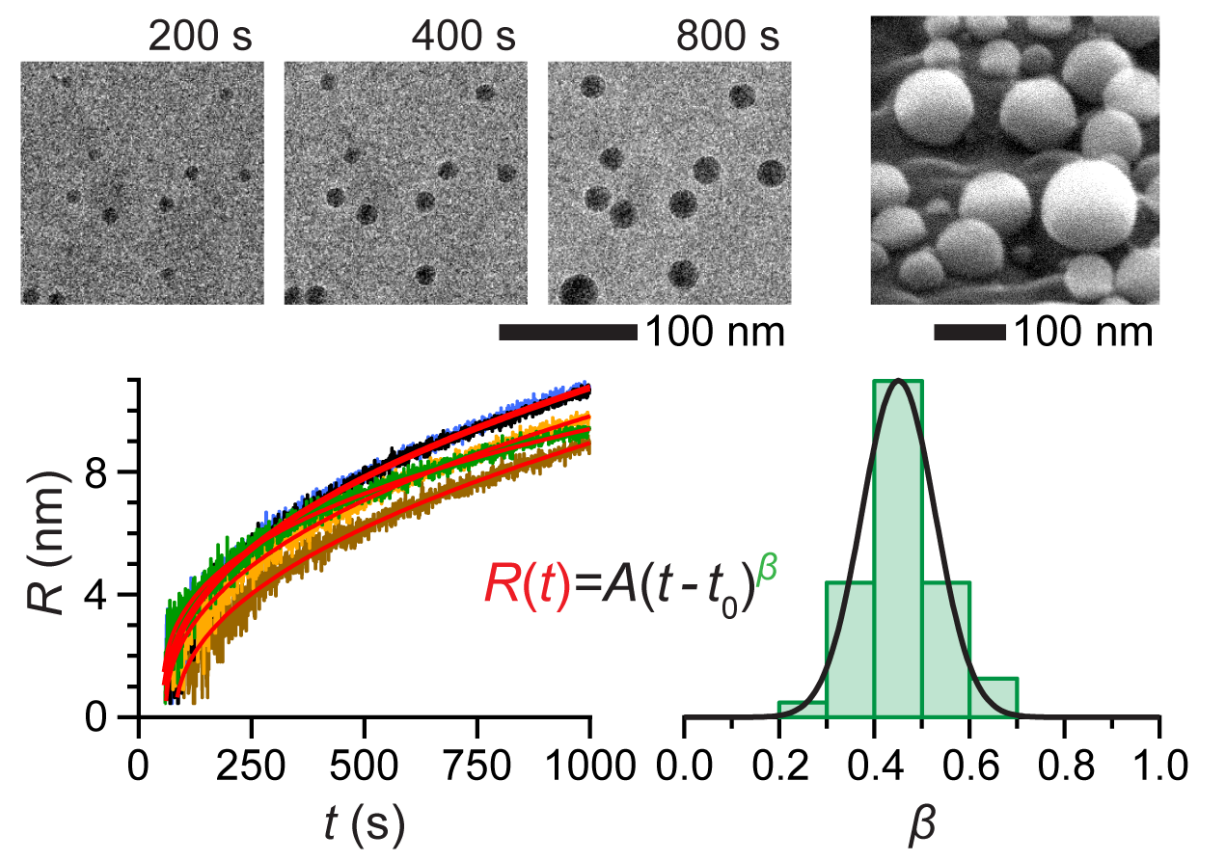

\begin{abstract}
The growth of catalytic liquid metal nanodroplets on flat substrates is essential for many technological applications. However, the detailed nucleation and growth dynamics of these nanodroplets remains unclear. Here, using in situ Transmission Electron Microscopy (TEM) imaging, we track in real-time the growth of individual Ga nanodroplets from a beam of Ga vapor. We show that the nucleation and growth are driven by thermally-activated surface diffusion of Ga adatoms, with the diffusion activation energy of $E_{\mathrm{D}}=95 \pm 10 \mathrm{meV}$ on a $\mathrm{SiN}_{\mathrm{x}}$ surface. More importantly, our
\end{abstract}


analysis shows that Ga-dimers serve as the critical nucleation clusters and that the nanodroplet growth follows a power-law of form $R(t) \propto e^{-E_{\mathrm{D}} / k_{\mathrm{B}} T}\left(t-t_{0}\right)^{1 / 2}$. These insights into the growth dynamics of metallic nanodroplets are essential for tailoring their size and density for their application in self-catalyzed growth of nanomaterials.

Liquid-metal nanoparticles have a wide range of applications in optics, ${ }^{1-2}$ catalysis, ${ }^{3}$ crystal growth, ${ }^{4}$ 2D materials growth ${ }^{5}$ and other areas of nanotechnology. ${ }^{6}$ The interest for these nanomaterials arises from their high surface reactivity and exceptional catalytic properties. For example, these nanodroplets act as excellent catalysts for the growth of semiconductor nanowires through vapor-liquid-solid (VLS) growth method, which is subject of great importance due to the variety of nanostructures that it enables to fabricate. ${ }^{7-9}$ Among various metals, Au nanodroplets are the most commonly used catalyst for the growth of group IV $^{10}$ and III-V nanowires. ${ }^{11-12}$ However, gold is not compatible with Si technology, ${ }^{13}$ and substitutes are required for the integration of nanowires into Si-based processes. In the case of III-V nanowires, the most promising alternative to Au-catalyzed growth is the self-catalyzed approach in which the group III constituent of the nanowire acts itself as a catalyst. In this method, the group III element is deposited on a substrate in a chemical vapor deposition or molecular beam epitaxy reactor to form droplets, ${ }^{14-15}$ and the group $\mathrm{V}$ element is added consecutively to promote the growth of nanowires. Therefore, it becomes essential to understand how the nanodroplets nucleate and grow on the surface, as their size will directly determine size ${ }^{16}$ and shape ${ }^{17}$ of the nanowire, thus affecting its bandgap energy ${ }^{18}$ which in turn affects the electronic ${ }^{19}$ and optical ${ }^{20}$ properties of the nanowires.

Despite the technological importance of liquid metal nanodroplets as catalysts, some aspects of their growth dynamics remain unknown. For example, it is not clear how these nanodroplets nucleate and grow as a function of time. The lack of this understanding stems from the challenge associated with the real-time observation of their growth processes. Previous characterization methods based on atomic force microscopy (AFM), ${ }^{21-22}$ scanning electron microscopy (SEM), ${ }^{14}$ scanning tunneling microscopy (STM), ${ }^{15}, 23-24$ low-energy electron microscopy (LEEM), ${ }^{25}$ and reflection high-energy electron diffraction (RHEED) ${ }^{26}$ are not suitable for tracking the evolution of many individual nanodroplets, and thus, cannot adequately describe the time-dependence of growth processes. Here, we use a modified transmission electron microscope (TEM) equipped with a Ga vapor beam source ${ }^{27}$ to observe in situ and in real-time the nucleation and growth dynamics of $\mathrm{Ga}$ nanodroplets on a $\mathrm{SiN}_{\mathrm{x}}$ substrate. In contrast to chemical precursors, which are prevalently used for in situ growth, ${ }^{28-}$ ${ }^{29}$ a Ga vapor beam does not require thermal decomposition to go into a liquid droplet. This ensures that the incident flux of material remains constant while the substrate temperature is changed, and it greatly improves the interpretability of the experimental results. From these results, we develop a model to describe the growth dynamics of Ga nanodroplets and show that 
their growth on surfaces follows a simple power-law, and it is dominated by thermally activated diffusion of $\mathrm{Ga}$ adatoms.
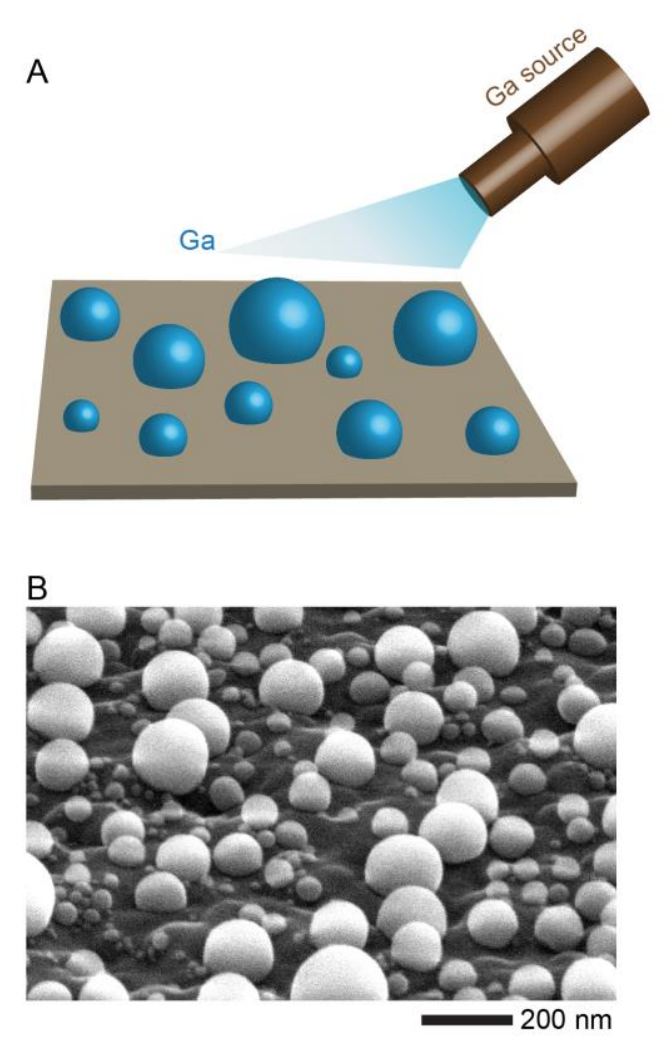
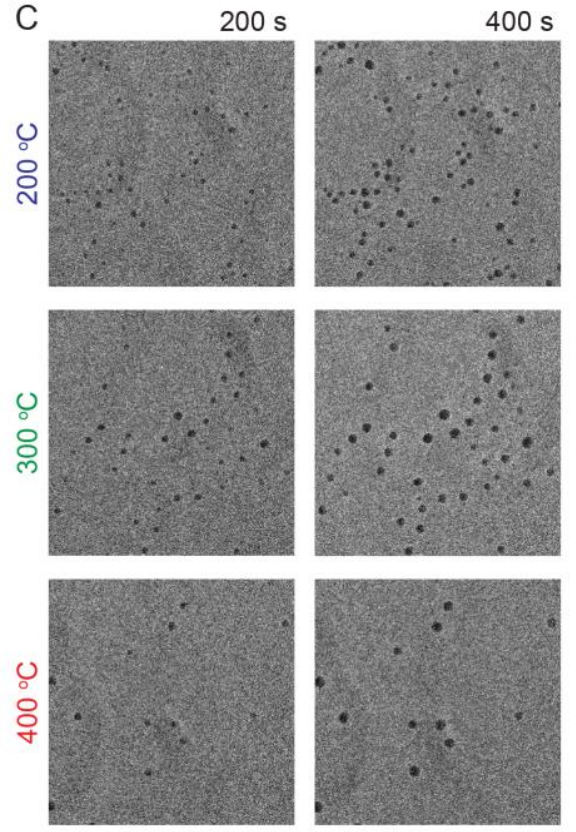

D
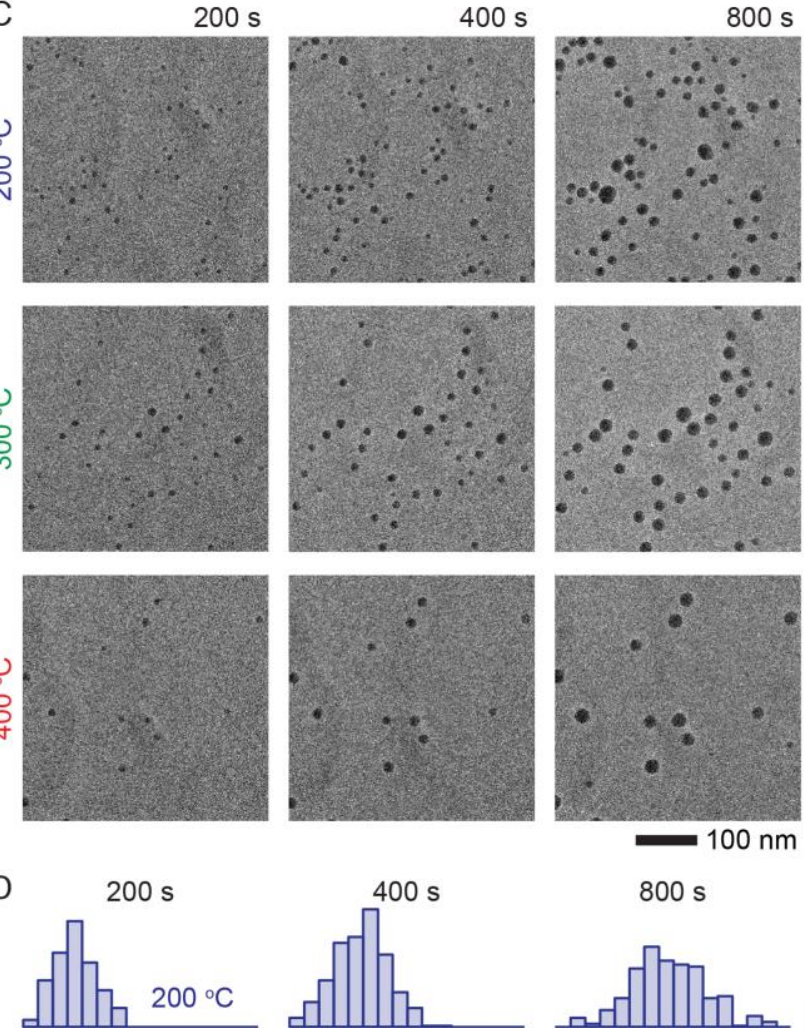

$800 \mathrm{~s}$
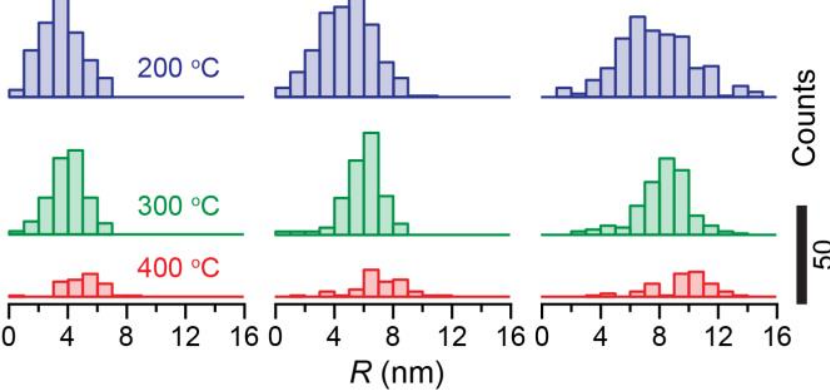

Figure 1. Overview of the growth dynamics of Ga nanodroplets. (A) Schematic representation of the experimental setup showing the growth of the Ga nanodroplets on a heated $\mathrm{SiN}_{\mathrm{x}}$ membrane from a Ga vapour beam source. (B) Scanning electron microscopy image of Ga nanodroplets on $\mathrm{SiN}_{\mathrm{x}}$ surface after Ga deposition. (C) Growth of $\mathrm{Ga}$ nanodroplets on a $\mathrm{SiN}_{\mathrm{x}}$ membrane held at $200{ }^{\circ} \mathrm{C}$ (Supplementary Video 1), $300{ }^{\circ} \mathrm{C}$ (Supplementary Video 2), and $400{ }^{\circ} \mathrm{C}$ (Supplementary Video 3). (D) Distribution of Ga nanodroplets at different times showing the evolution of the nanodroplet radii distributions at $200{ }^{\circ} \mathrm{C}$ (blue), $300{ }^{\circ} \mathrm{C}$ (green), and $400{ }^{\circ} \mathrm{C}$ (red).

Figure 1A shows the experimental schematic used for imaging the growth dynamics of $\mathrm{Ga}$ nanodroplets. The nanodroplets were grown on a flat 50 -nm-thick $\mathrm{SiN}_{\mathrm{x}}$ membrane held at different temperatures $\left(200{ }^{\circ} \mathrm{C}, 250{ }^{\circ} \mathrm{C}, 300{ }^{\circ} \mathrm{C}, 350{ }^{\circ} \mathrm{C}, 400{ }^{\circ} \mathrm{C}, 450{ }^{\circ} \mathrm{C}, 500{ }^{\circ} \mathrm{C}\right)$ inside the TEM. The arriving Ga flux was controlled by the temperature of the thermal effusion cell, which was held at a fixed temperature of $940{ }^{\circ} \mathrm{C}$. Figure 1B shows the post-growth SEM image of typical nanodroplets on the membrane. The nucleation and growth dynamics of the nanodroplets on the $\mathrm{SiN}_{\mathrm{x}}$ substrate held at temperatures of $200{ }^{\circ} \mathrm{C}, 300{ }^{\circ} \mathrm{C}, 400{ }^{\circ} \mathrm{C}$ (Figure $1 \mathrm{C}$ D, Supplementary Videos 1-3) reveals that nucleation occurs at early stages of Ga deposition, 
after which the droplets mostly grow in size with very few nucleation events taking place at $t$ $>200$ s. Also, the droplet density on the surface decreases with the increasing surface temperature (Figure 1C-D).
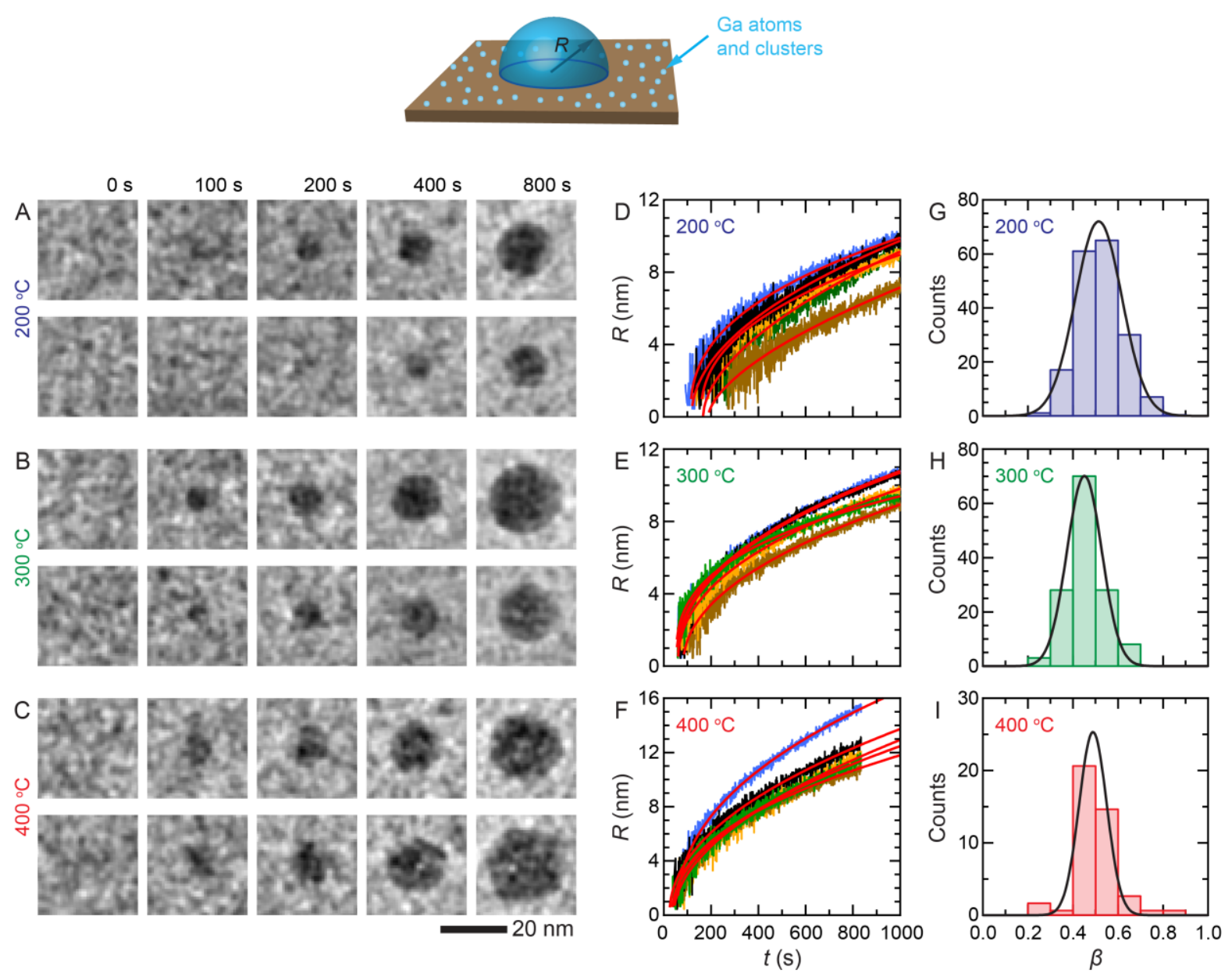

Figure 2. Growth dynamics of Ga nanodroplets at different substrate temperatures. A time series of in situ TEM images showing the nucleation and growth of Ga nanodroplets at a substrate temperature of $(\mathbf{A}) T=200^{\circ} \mathrm{C}$, (B) $300{ }^{\circ} \mathrm{C}$, and $(\mathbf{C}) 400{ }^{\circ} \mathrm{C}$. The radii of five representative nanodroplets as a function of time at $(\mathbf{D}) T=200{ }^{\circ} \mathrm{C}$, (E) $T=300{ }^{\circ} \mathrm{C}$, and $(\mathbf{F}) T=400{ }^{\circ} \mathrm{C}$. The solid red lines represent the power law fit to $R(t) \sim\left(t-t_{0}\right)^{\beta}$. (G-I) Histograms depicting the distribution of the exponents $(\beta)$ from the power-law fits yields $\beta$ of $0.51 \pm 0.07,0.45 \pm$ 0.05 , and $0.49 \pm 0.04$ for the substrate temperatures of $T=200{ }^{\circ} \mathrm{C}, T=300{ }^{\circ} \mathrm{C}$, and $T=400{ }^{\circ} \mathrm{C}$, respectively.

To reveal the underlying mechanisms of the growth, we tracked the evolution of more than $600 \mathrm{Ga}$ nanodroplets at different substrate temperatures. Figure $2 \mathrm{~A}-\mathrm{C}$ show the nucleation and growth of individual Ga droplets occurring at three different substrate temperatures (for growth trajectories at other substrate temperatures see Supporting Information Section 1). Here, the plot of the nanodroplet radii as a function of time (Figure 2D-F) shows that the growth behavior is similar in all the cases (i.e., curve shapes are similar) despite the different substrate temperatures. 
To describe the observed growth dynamics, we first consider that the nanodroplet nucleation on the $\mathrm{SiN}_{\mathrm{x}}$ substrate occurs when a critical number of $\mathrm{Ga}$ adatoms form a critical cluster. These clusters form by agglomeration of adatoms driven by their surface diffusion. ${ }^{30}$ The subsequent steady-state growth of the nanodroplets is sustained through the continuous deposition of Ga onto the substrate. ${ }^{31}$ The observed growth process can be explained through a simple model. We assume the shape of the nanodroplet, which nucleated at any arbitrary time $t=t_{0}$, to be a hemisphere (Figure 2, top). The growth of the nanodroplet volume comes from two sources: $i$ ) the direct deposition of Ga from vapor at the vapor-liquid interface and $i i$ ) the surface flux of diffusing $\mathrm{Ga}$ adatoms on a substrate at the triple phase contact line. The growth rates of the nanodroplet volume $\left(V=2 \pi R^{3} / 3\right)$ for the first and second cases are given by:

$$
\frac{\mathrm{d} V}{\mathrm{~d} t}=2 \pi R^{2} \frac{\mathrm{d} R}{\mathrm{~d} t} \propto \pi R^{2} F
$$

and

$$
\frac{\mathrm{d} V}{\mathrm{~d} t}=2 \pi R^{2} \frac{\mathrm{d} R}{\mathrm{~d} t} \propto 2 \pi R S
$$

respectively. Here, $F$ is the Ga arrival rate (volumetric flux), and $S$ is the surface flux of the diffusing $\mathrm{Ga}$ adatoms at the triple-phase contact line. For simplicity of the discussion and analysis, we assume that the surface flux, whose dimension is equivalent to volume flux per unit of length, is constant over time and independent of the size of the nanodroplet. The solution to these simple ordinary differential equations is:

$$
R(t)=A\left(t-t_{0}\right)^{\beta}
$$

where the exponent $\beta$ is 1 for the growth by the adsorption of $\mathrm{Ga}$ vapor directly to the nanodroplet surface (Eq. 1) and $1 / 2$ for the growth through adatom flux at the contact line (Eq. 2 ). Generally, for the case when both growth modes are present, the $\beta$ exponent will be in the range between $1 / 2$ and 1 . Moreover, note that even though we assumed the droplet to have a hemispherical shape, the same power-law behavior is true for droplets with any spherical cap shapes (Supporting Information Section 2).

Remarkably, the fit of the droplet radii to a power-law $R(t)=A\left(t-t_{0}\right)^{\beta}$ (Figure 2DF) results in the exponents of $\beta \approx 1 / 2$, which are in a good agreement with the growth driven by Ga flux towards the triple-phase-contact line. This supports our initial hypothesis that the main contribution to the droplet growth is from the flux of $\mathrm{Ga}$ adatoms at the $\mathrm{SiN}_{\mathrm{x}}$ surface. The values of the exponents obtained for all the nanodroplets at three different substrate temperatures are summarized in the histograms shown in Figure 2G-I (for the fits to the growth trajectories at other substrate temperatures and corresponding histograms see Supporting Information Section 1). Note that in the case of micro- and macroscopic droplets, we expect the growth to be dominated by the adsorption of Ga directly to a nanodroplet surface during 
the deposition, and the growth to be linear with time $\left(R(t) \sim\left(t-t_{0}\right)\right.$ ) (i.e., $\beta \approx 1$ ) (Supporting Information Section 2).

The nanodroplet growth rates increase with temperature (Figure 2D-F), indicating an increase in adatom flux $(S)$ towards the nanodroplet. ${ }^{32}$ Under the steady-state condition, the flux is proportional to the adatom diffusion coefficient, ${ }^{33}$ and the observed increase in the flux is consistent with the expected increase in the diffusivity: ${ }^{15}$

$$
D=D_{0} \exp \left(E_{\mathrm{D}} / k_{\mathrm{B}} T\right)
$$

Here, $D_{0}, k_{\mathrm{B}}, E_{\mathrm{D}}$, and $T$ are diffusion prefactor, Boltzmann constant, an activation energy barrier for the diffusion, and the substrate temperature, respectively. The activation energy for the surface diffusion can be extracted directly from the fits to the growth curves. To estimate the activation energy, we first calculated the average flux at different temperatures by fitting all the nanodroplet growth curves to Eq. 3 with $\beta=1 / 2$ (Figure 3A). Note that from Eqs. 2-3, we have $A^{2} \propto S$. Next, by fitting the temperature-dependent average flux values to Eq. 4 (Figure 3B), we readily obtain the diffusion activation energy barrier of $E_{\mathrm{D}}=95 \pm 10 \mathrm{meV}$ for $\mathrm{Ga}$ adatoms on an amorphous $\mathrm{SiN}_{\mathrm{x}}$ surface. For comparison, this value is much smaller than the activation energy of $1.7 \mathrm{eV}$ for the diffusion of $\mathrm{Ga}$ on GaAs surfaces, ${ }^{23}$ where the $\mathrm{Ga}$ adatoms are known to chemically bond with the surface. ${ }^{34}$ Hence, the low activation energy obtained from our results suggests the absence of any bonding with the surface, and Ga adatoms are likely to be only physisorbed to the $\mathrm{SiN}_{\mathrm{x}}$ surface.
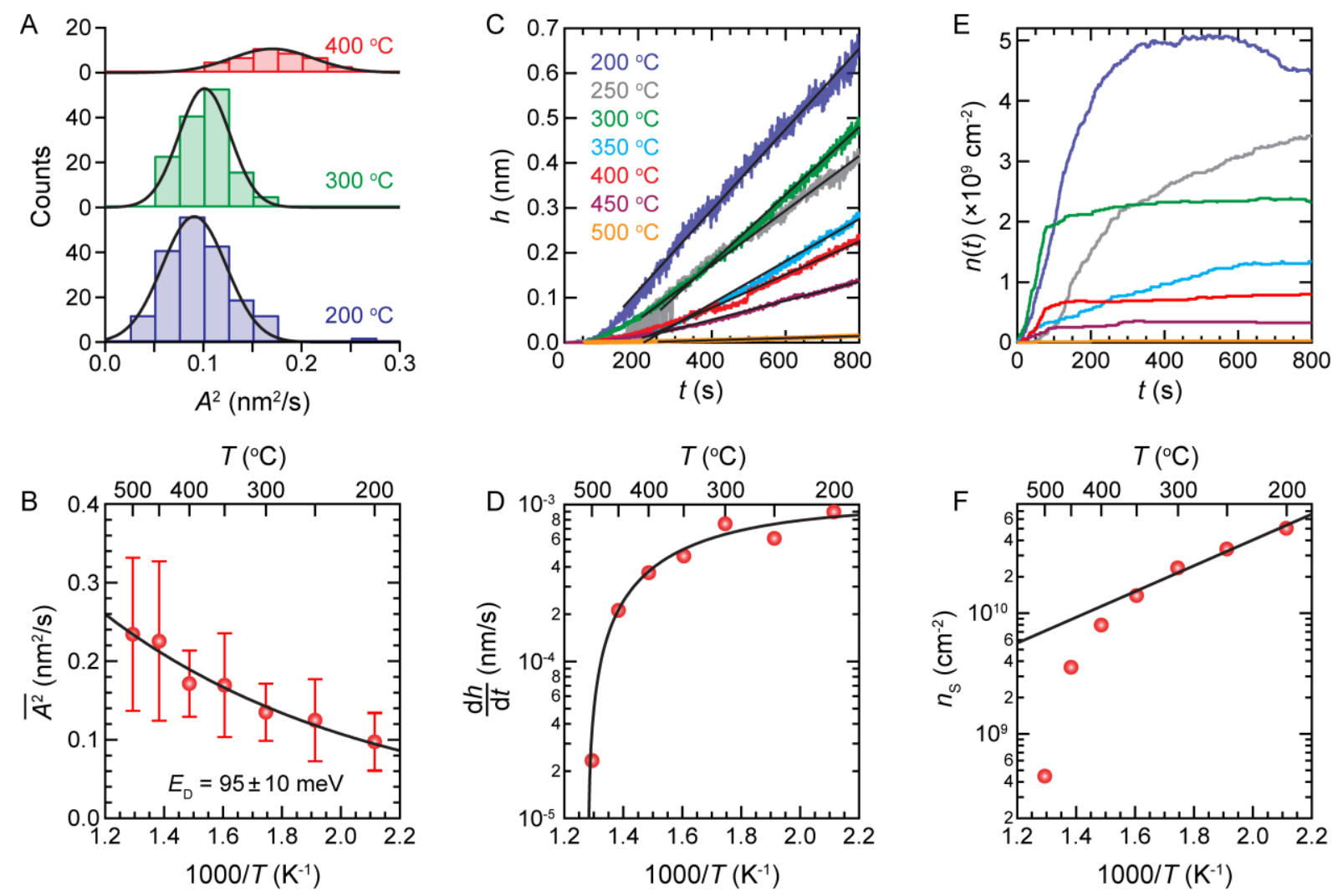
Figure 3. Density of Ga nanodroplets at different substrate temperatures. (A) Distribution of the calculated flux contributing to the growth of each nanodroplet at $200{ }^{\circ} \mathrm{C}$ (blue), $300{ }^{\circ} \mathrm{C}$ (green), and $400{ }^{\circ} \mathrm{C}$ (red). The values of $\mathrm{Ga}$ adatom flux are obtained by fitting the nanodroplet growth curves to Eq. 2. Black solid lines are the Gaussian fits to the histograms. (B) Plot of the average adatom flux for all the droplets versus the surface temperature. The error bars represent one standard deviation. The activation diffusion energy of $E_{\mathrm{a}}=95 \pm 10$ is obtained from the exponential fit (Eq. 3) plotted as a black curve. (C) Effective Ga thickness deposited on a $\mathrm{SiN}_{\mathrm{x}}$ substrate held at different temperatures as a function of time. Thickness was estimated from the volume of all the nanodroplets forming in the field of view $\left(\sim 0.6-0.7 \mu \mathrm{m}^{2}\right)$. Here, droplets are assumed to be hemispherical. Black lines represent the linear fit to the data used to estimate the growth rate. (D) Ga deposition rate plotted as a function of the substrate temperature. Black solid curve is a fit to Eq. 4, from which the desorption activation energy of $E_{\mathrm{d}}=$ $202 \pm 116 \mathrm{meV}$ is extracted. (E) The density of Ga nanodroplets in the field of view as a function of time for the growth at different substrate temperatures. Colors correspond to the same temperatures as in (C). (F) The saturation nucleation density of the nanodroplets versus the substrate temperature. The critical cluster (dimer) formation energy of $E_{2}=775 \pm 127 \mathrm{meV}$ is extracted by fitting (black solid line) the data points at $T \leq 350{ }^{\circ} \mathrm{C}$ to the complete-condensation model (Eq. 6).

Apart from diffusion and nucleation, $\mathrm{Ga}$ adatoms moving on the flat surface also experience desorption. After the nucleation saturates (i.e., very few new nanodroplets are forming) and the system reaches a steady-state regime, the amount of Ga adatoms consumed for the nucleation of new clusters is insignificant. Hence, the desorption process can be quantified based on the growth of stable nanodroplets. The effective thickness of Ga estimated by dividing the summed volume of all the hemispherical nanodroplets by the area of the field of view is plotted in Figure 3C. Note that the arrival rate of $\mathrm{Ga}$ onto the $\mathrm{SiN}_{\mathrm{x}}$ substrate was the same during the in situ TEM imaging experiments at all seven substrate temperatures. The growth rates of the effective Ga thickness obtained from the slope of the linear fits in Figure $3 \mathrm{C}$ show that the growth rate decreases drastically for high substrate temperatures $(T>350$ $\left.{ }^{\circ} \mathrm{C}\right)$. Here, we emphasize that the evaporation of $\mathrm{Ga}$ from the nanodroplets is negligible for all the substrate temperatures used for our study $\left(T \leq 500{ }^{\circ} \mathrm{C}\right)$, and the evaporation becomes relevant only at $T \geq 550{ }^{\circ} \mathrm{C}$ (Supporting Information Section 3). To describe the Ga desorption process, we approximated the growth rate as the difference between the arrival rate of Ga onto a surface and desorption from the surface: ${ }^{31}$

$$
\frac{\mathrm{d} h}{\mathrm{~d} t}=F-B \exp \left(-E_{\mathrm{d}} / k_{\mathrm{B}} T\right)
$$

Here, the second term is the desorption rate where $E_{\mathrm{d}}$ is the activation energy barrier for the desorption. From the fit of Eq. 5 to the data in Figure 3D, we obtain the Ga arrival rate of $F=$ $0.59 \pm 0.15 \AA / \mathrm{min}$, and the desorption activation energy is $E_{\mathrm{d}}=202 \pm 116 \mathrm{meV}$. The lower activation energy for $\mathrm{Ga}$ desorption from $\mathrm{SiN}_{\mathrm{x}}$ than from the $\mathrm{GaAs}$ surface of $2.5 \mathrm{eV}$ is consistent with our earlier assertion about Ga being physisorbed to the $\mathrm{SiN}_{\mathrm{x}}$ membrane surface during the deposition. ${ }^{35}$ Note that the sharp increase in the desorption rate at $T \geq 350{ }^{\circ} \mathrm{C}$ (Figure 3C) and the corresponding decrease in the steady-state concentration of $\mathrm{Ga}$ at the surface should lower the nucleation rate. 
To put these observations in the context of nanowire growth, we note that semiconductor nanowires are usually grown at higher temperatures, ${ }^{11}$ at which $\mathrm{Ga}$ has high desorption rate and the nucleation density of the nanodroplets is low. Controlling the desorption rate is important for the growth of nanowires. In particular, on pre-patterned substrates where positions of nanowires are determined by the patterning mask, the desorption of Ga from the surface regulates the amount of Ga deposited on the nanowires. ${ }^{36-37}$ Thus, the desorption activation energy is essential for choosing the fluxes of materials used for MBE growth at different temperatures.

The density of Ga nanodroplets as a function of time at different temperatures (Figure $3 \mathrm{E})$ shows that the nucleation events mostly take place early $(t<200 \mathrm{~s})$ and saturate afterward. The saturation nucleation density decreases with an increase in the substrate temperature (Figure 3F). As discussed earlier, Ga desorption becomes significant at elevated temperatures $\left(T \geq 350{ }^{\circ} \mathrm{C}\right.$ ) (Figure $\left.3 \mathrm{C}-\mathrm{D}\right)$. For simplicity, at a lower temperature $\left(T \leq 300{ }^{\circ} \mathrm{C}\right)$, we assume a complete $\mathrm{Ga}$ condensation on the surface. According to mean-field nucleation theory for complete condensation processes (i.e., no adatom desorption from the substrate), the saturation density for $3 \mathrm{D}$ islands is given by: ${ }^{38}$

$$
n_{\mathrm{S}} \sim F^{i} \exp \left(\frac{i E_{\mathrm{D}}+E_{i}}{(i+2.5) k_{\mathrm{B}} T}\right)
$$

Here, $E_{i}$ is the critical cluster formation energy for a cluster of $i$ atoms (i.e., the cluster with $i+1$ atoms is the smallest stable cluster). Note that the activation energy for the surface diffusion is $E_{\mathrm{D}}=95 \mathrm{meV}$ (Figure 3B). At low substrate temperatures, the critical cluster is expected to be the Ga dimer $(i=2) .{ }^{39}$ Then by fitting the saturation nucleation density at $T \leq 350{ }^{\circ} \mathrm{C}$ to Eq. 6 , we obtain the cluster formation energy of $E_{2}=775 \pm 127 \mathrm{meV}$ (Figure 3F). This value is consistent with the Ga-dimer energy of $1.1 \mathrm{eV},{ }^{40}$ indicating that critical cluster for the nanodroplet nucleation on our experiments is likely a Ga dimer.

So far, the growth dynamics discussed here was based on the interactions of an isolated nanodroplet with $\mathrm{Ga}$ vapor and the flat surface. However, potential interactions between neighboring nanodroplets might also alter the growth dynamics and statistics. For example, at $T \geq 350{ }^{\circ} \mathrm{C}$, the nanodroplet density deviates drastically from the fit (Figure 3F), consistent with other studies at elevated temperatures. ${ }^{24,39,41}$ There are several reasons for such a deviation. First, as mentioned earlier, Eq. 6 does not account for desorption of the Ga adatoms, which becomes significant at high temperatures (Figure 3C-D). Second, at high temperatures, the diffusive movement of clusters may also contribute to the subsequent coalescence and Ostwald ripening of small Ga clusters reducing the number of nanodroplets on the surface. Detecting the interaction dynamics between these small clusters is beyond our current experimental resolution, and we can only detect the droplets that are large enough ( $22 \mathrm{~nm})$. Hence, in order to show the mechanism of Ostwald ripening and coalescence, we studied these 
processes in detail at lower temperatures, when the slower diffusion of adatoms allows the merging clusters to grow up to detectable sizes.

Examples of coalescence and Ostwald ripening of nanodroplets that are $\gtrsim 2 \mathrm{~nm}$ are shown in Figure 4. We found that the frequency of these events depends on the proximity of the nanodroplets to each other, and increases with an increase in a nanodroplet density (i.e., lower substrate temperatures, Figure 3F). For example, at a substrate temperature of $200{ }^{\circ} \mathrm{C}$, 121 and 7 out of 280 nanodroplets undergo coalescence and Ostwald ripening, respectively, whereas this number drops to 1 and 0 out of 51 nanodroplets for the growth studies at $400{ }^{\circ} \mathrm{C}$. The gradual decrease in the nanodroplet density at $t>600 \mathrm{~s}$ seen in the nucleation curve at a substrate temperature of $200{ }^{\circ} \mathrm{C}$ (Figure 3E) is due to these coarsening processes. These observations show yet another growth mode through which nanodroplets grow in size, which is similar to the nanoparticle growth from solution. ${ }^{42}$ However, at high temperatures, the coalescence between small mobile clusters, which are below our detection threshold, is expected to dominate, consuming the nanodroplets before they become detectable. The observed low droplet density at high temperature is also consistent with this explanation.
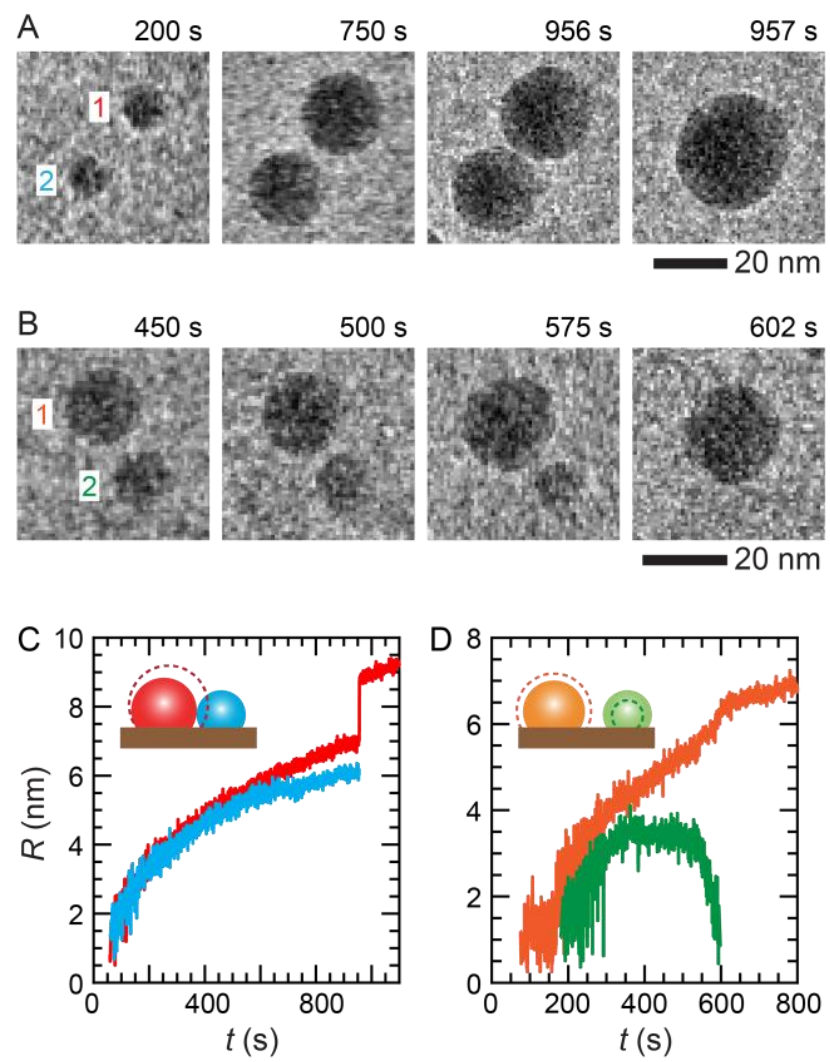

Figure 4. Coalescence and Ostwald ripening of Ga nanodroplets. A time series TEM images showing (A) the coalescence and (B) Ostwald ripening between two Ga nanodroplets at $300{ }^{\circ} \mathrm{C}$ and $250{ }^{\circ} \mathrm{C}$, respectively. Radii of the nanodroplets $(\mathbf{C})$ undergoing coalescence and (D) Ostwald ripening as shown in (A) and (B), respectively.

Generally, two of the most widely used materials for masking deposition of Ga are $\mathrm{SiN}_{\mathrm{x}}$, and $\mathrm{SiO}_{2}$. Based on the previous ex situ nucleation studies, ${ }^{14,32}$ we expect the two 
substrates to behave similarly, though relevant activation energies may slightly vary between these two amorphous surfaces. The situation is expected to be different for Ga nanodroplets forming on a crystalline surface. ${ }^{43}$ The model of the nanodroplet growth is not only limited to $\mathrm{Ga}$ but can be extended to other liquid-metal nanodroplets whose growth on a substrate is dominated by the surface flux of adatoms.

In summary, our observations reveal that the thermally activated diffusion of the adatoms drive the nucleation and growth of $\mathrm{Ga}$ nanodroplets, and the nanodroplet growth follows a power-law: $R(t) \sim e^{-E_{\mathrm{D}} / k_{\mathrm{B}} T}\left(t-t_{0}\right)^{1 / 2}$. Furthermore, the observed decrease of the nanodroplet density with increase in substrate temperature suggests a strategy for controlling the density of catalytic nanodroplets during the deposition process. In particular, for nanowire growth, our study provides an approach to fine-tune their density and size by revealing key parameters that control the growth of catalytic nanodroplet. More generally, our study has broad implications for the growth of wide-range of metallic nanocatalysts and other nanomaterials on surfaces.

\section{Supporting Information}

The following files are available free of charge.

Supplementary text and figures discussing additional experiments, analyses, and modeling (PDF).

Growth of $\mathrm{Ga}$ nanodroplets at a substrate temperature of $200{ }^{\circ} \mathrm{C}$. Segmented droplet boundaries are highlighted in white (AVI).

Growth of $\mathrm{Ga}$ nanodroplets at a substrate temperature of $300{ }^{\circ} \mathrm{C}$. Segmented droplet boundaries are highlighted in white (AVI).

Growth of $\mathrm{Ga}$ nanodroplets at a substrate temperature of $400{ }^{\circ} \mathrm{C}$. Segmented droplet boundaries are highlighted in white (AVI).

\section{Acknowledgments}

This work was supported by the Singapore National Research Foundation's Competitive Research Program funding (NRF-CRP16-2015-05). We also acknowledge the "Agence Nationale de la Recherche", the French funding agency, for funding the NanoMAX ETEM through the "Equipements d'excellence" TEMPOS, Project No. 10-EQPX-0050. We acknowledge Odile Stéphan and Jean-Luc Maurice for their continuous support and Ileana Florea for technical assistance during the ETEM experiments. 


\section{Materials and Methods}

Experimental Procedures: For imaging the growth of Ga nanodroplets, we used Titan ETEM, which is a prototype TEM built by Thermo Fisher (Waltham, MA, USA) as part of the NanoMAX project. It operates at $300 \mathrm{keV}$, and the image is corrected from spherical aberrations. This ETEM is designed to accommodate MBE effusion cell sources developed at C2N (Palaiseau, France). One of the cells was loaded with solid Ga (99.9999\% purity) and heated to $940{ }^{\circ} \mathrm{C}$ to generate a Ga vapor beam directed toward the sample holder. The operating temperature was optimized by monitoring the density of the Ga droplets at different effusion cell temperatures (Supporting Information Section 4). The vapor beam can be stopped by a shutter, and the flux is adjusted by changing the temperature of the source. The background pressure in the object chamber is $8 \times 10^{-7} \mathrm{~Pa}$; this value is not affected when beams of matter are supplied to the sample. The substrate consisted of a $\mathrm{SiN}_{\mathrm{x}}$ film deposited over a $\mathrm{SiC}$ heating membrane (Protochip Inc., Morrisville, NC, USA). The modified Protochips Fusion TEM holder allows a line of sight between the collimated beam flux and the $\mathrm{SiN}_{\mathrm{x}}$ substrate. The movies were captured with electron flux ranging from 30 to $40 \mathrm{e}^{-} /\left(\AA^{2} \cdot \mathrm{s}\right)$ at a rate of 4 frames per second with the US1000 CCD camera (Gatan, Inc., Pleasanton, CA, USA).

Image Processing and data analysis: The segmentation algorithm was written in Python- $2.7^{44}$ with the implementation of the following libraries, such as numpy, ${ }^{45}$ scipy, ${ }^{46}$ OpenCV, ${ }^{47}$ scikit-image, ${ }^{48}$ cython, ${ }^{49}$ and matplotlib. ${ }^{50}$ The recorded movies were saved as a sequence of 8-bit grayscale images. These raw images were then inverted, and a low-pass Gaussian filter with $\sigma=3$ was applied on the inverted images in order to eliminate their highfrequency noise. It is essential to point out that the image background was not uniform, and also the nanodroplets could be situated close to each other, which means that the conventional top-hat transform ${ }^{51}$ was not applicable for this case. In order to address these challenges, we applied the Rotational Morphological Processing (RMP) to the inverted blurred images. ${ }^{52}$ RMP performs grayscale morphological opening and utilizes a 1-pixel wide rod as the structuring element, whereas the length of the rod must be bigger than the maximum size of the object. However, as larger structuring element would require longer computational time, we gradually varied the length of the rod, typically, from 25 pixels in the beginning, when all nanodroplets were very small, to 75 pixels at the end of the movie. For full RMP procedure, each image was rotated from $0^{\circ}$ to $360^{\circ}$ with a step of $1^{\circ}$, and the corresponding background-subtracted images were generated and stacked together. The stack was used to find the total background, which is defined as maximum for all slices in the stack. Subsequently, the final result of the RMP refined image - was acquired as the difference between the inverted blurred image and the total background. Then refined images were used to generate binary images by applying intensitybased Kapur threshold algorithm. ${ }^{53}$

After obtaining the binary images, the nanodroplets were labeled based on their size and position. Since we detected more false positives at the beginning of each movie when the contrast is weak due to a small size and low density of the nanodroplets, we labeled the 
nanodroplets starting from the last frame, when all nanodroplets are bigger and can be easily segmented. Subsequently, we filtered the false positives out based on their occurrence and maximum radius defined as $r=\sqrt{A_{\text {nanodrop }} / \pi}$, where $A_{\text {nanodrop }}$ is the maximum area of the nanodroplet. 
Figure Captions:

Figure 1. Overview of the growth dynamics of Ga nanodroplets. (A) Schematic representation of the experimental setup showing the growth of the Ga nanodroplets on a heated $\mathrm{SiN}_{\mathrm{x}}$ membrane from a Ga vapor beam source. (B) Scanning electron microscopy image of $\mathrm{Ga}$ nanodroplets on $\mathrm{SiN}_{\mathrm{x}}$ surface after Ga deposition. (C) Growth of Ga nanodroplets on a $\mathrm{SiN}_{\mathrm{x}}$ membrane held at $200{ }^{\circ} \mathrm{C}$ (Supplementary Video 1), $300{ }^{\circ} \mathrm{C}$ (Supplementary Video 2), and $400{ }^{\circ} \mathrm{C}$ (Supplementary Video 3). (D) Distribution of $\mathrm{Ga}$ nanodroplets at different times showing the evolution of the nanodroplet radii distributions at $200{ }^{\circ} \mathrm{C}$ (blue), $300{ }^{\circ} \mathrm{C}$ (green), and $400{ }^{\circ} \mathrm{C}$ (red).

Figure 2. Growth dynamics of Ga nanodroplets at different substrate temperatures. Time series of in situ TEM images showing the nucleation and growth of Ga nanodroplets at substrate temperatures of (A) $200{ }^{\circ} \mathrm{C}$, (B) $300{ }^{\circ} \mathrm{C}$, and (C) $400{ }^{\circ} \mathrm{C}$. The radii of five representative nanodroplets as a function of time at $(\mathbf{D}) 200{ }^{\circ} \mathrm{C}$, (E) $300{ }^{\circ} \mathrm{C}$, and $(\mathbf{F}) 400{ }^{\circ} \mathrm{C}$. The red curves represent the power-law fit to $R(t) \propto\left(t-t_{0}\right)^{\beta}$. (G-I) Histograms depicting the distribution of the exponents $(\beta)$ from the power-law fits yield $\beta$ of $0.51 \pm 0.07,0.45 \pm 0.05$, and $0.49 \pm 0.04$ for the substrate temperatures of $200{ }^{\circ} \mathrm{C}, 300{ }^{\circ} \mathrm{C}$, and $400{ }^{\circ} \mathrm{C}$, respectively.

Figure 3. Analysis of nucleation and growth parameters of Ga nanodroplets at different substrate temperatures. (A) Distribution of the calculated flux contributing to the growth of each nanodroplet at $200{ }^{\circ} \mathrm{C}$ (blue), $300{ }^{\circ} \mathrm{C}$ (green), and $400{ }^{\circ} \mathrm{C}$ (red). The values of Ga adatom flux were obtained by fitting the nanodroplet growth curves to Eq. 3. Black curves are the Gaussian fits to the histograms. (B) Plot of the average adatom flux for all the nanodroplets versus the substrate temperature. The error bars represent one standard deviation. The activation diffusion energy of $E_{\mathrm{a}}=95 \pm 10$ was obtained from the exponential fit (Eq. 4) plotted as a black curve. (C) Effective Ga thickness deposited on a $\mathrm{SiN}_{\mathrm{x}}$ substrate held at different temperatures as a function of time. Thickness was estimated from the volume of all the nanodroplets forming in the field of view $\left(\sim 0.6-0.7 \mu \mathrm{m}^{2}\right)$. Here, the nanodroplets are assumed to be hemispherical. Black lines represent the linear fit to the data used to estimate the growth rate. (D) Ga deposition rate plotted as a function of the substrate temperature. Black curve is a fit to Eq. 5, from which the desorption activation energy of $E_{\mathrm{d}}=202 \pm 116 \mathrm{meV}$ was extracted. (E) The density of Ga nanodroplets in the field of view as a function of time for the growth at different substrate temperatures. Colors correspond to the same temperatures as in (C). (F) The saturation nucleation density of the nanodroplets versus the substrate temperature. The critical cluster (dimer) formation energy of $E_{2}=775 \pm 127 \mathrm{meV}$ was extracted by fitting the complete-condensation model (Eq. 6) (black line) to the data points at $T \leq 350{ }^{\circ} \mathrm{C}$.

Figure 4. Coalescence and Ostwald ripening of Ga nanodroplets. Time series of in situ TEM images showing (A) the coalescence and (B) Ostwald ripening between two Ga nanodroplets at $300{ }^{\circ} \mathrm{C}$ and $250{ }^{\circ} \mathrm{C}$, respectively. Radii of the nanodroplets undergoing $(\mathbf{C})$ coalescence and (D) Ostwald ripening as shown in (A) and (B), respectively. 


\section{References}

1. Losurdo, M.; Yi, C.; Suvorova, A.; Rubanov, S.; Kim, T.-H.; Giangregorio, M. M.; Jiao, W.; Bergmair, I.; Bruno, G.; Brown, A. S. Demonstrating the capability of the high-performance plasmonic gallium-graphene couple. ACS Nano 2014, 8 (3), 3031-3041.

2. Gurioli, M.; Wang, Z.; Rastelli, A.; Kuroda, T.; Sanguinetti, S. Droplet epitaxy of semiconductor nanostructures for quantum photonic devices. Nat. Mater. 2019, 18 (8), 799-810.

3. Taccardi, N.; Grabau, M.; Debuschewitz, J.; Distaso, M.; Brandl, M.; Hock, R.; Maier, F.; Papp, C.; Erhard, J.; Neiss, C. Gallium-rich Pd-Ga phases as supported liquid metal catalysts. Nat. Chem. 2017, 9 (9), 862.

4. Wang, F.; Dong, A.; Buhro, W. E. Solution-liquid-solid synthesis, properties, and applications of one-dimensional colloidal semiconductor nanorods and nanowires. Chem. Rev. 2016, 116 (18), 10888-10933.

5. Chen, Y.; Liu, K.; Liu, J.; Lv, T.; Wei, B.; Zhang, T.; Zeng, M.; Wang, Z.; Fu, L. Growth of 2D GaN Single Crystals on Liquid Metals. J. Am. Chem. Soc. 2018, 140 (48), 16392-16395.

6. Wang, Z. M. Nanodroplets. Springer: 2014; Vol. 18.

7. Dick, K. A.; Deppert, K.; Larsson, M. W.; Mårtensson, T.; Seifert, W.; Wallenberg, L. R.; Samuelson, L. Synthesis of branched 'nanotrees' by controlled seeding of multiple branching events. Nat. Mater. 2004, 3 (6), 380.

8. Tian, B.; Xie, P.; Kempa, T. J.; Bell, D. C.; Lieber, C. M. Single-crystalline kinked semiconductor nanowire superstructures. Nat. Nanotechnol. 2009, 4 (12), 824.

9. Panciera, F.; Chou, Y.-C.; Reuter, M.; Zakharov, D.; Stach, E.; Hofmann, S.; Ross, F. Synthesis of nanostructures in nanowires using sequential catalyst reactions. Nat. Mater. 2015, 14 (8), 820.

10. Amato, M.; Palummo, M.; Rurali, R.; Ossicini, S. Silicon-germanium nanowires: chemistry and physics in play, from basic principles to advanced applications. Chem. Rev. 2013, 114 (2), 1371-1412.

11. Güniat, L.; Caroff, P.; Fontcuberta i Morral, A. Vapor Phase Growth of Semiconductor Nanowires: Key Developments and Open Questions. Chem. Rev. 2019. (DOI: 10.1021/acs.chemrev.8b00649)

12. Fan, H. J.; Werner, P.; Zacharias, M. Semiconductor nanowires: from self-organization to patterned growth. Small 2006, 2 (6), 700-717.

13. Kang, J.; Schroder, D. Gettering in silicon. J. Appl. Phys. 1989, 65 (8), 2974-2985.

14. Detz, H.; Kriz, M.; MacFarland, D.; Lancaster, S.; Zederbauer, T.; Capriotti, M.; Andrews, A. M.; Schrenk, W.; Strasser, G. Nucleation of Ga droplets on Si and SiO x surfaces. Nanotechnology 2015, 26 (31), 315601.

15. Brune, H. Microscopic view of epitaxial metal growth: nucleation and aggregation. Surf. Sci. Rep. 1998, 31 (4), 125-229.

16. Cui, Y.; Lauhon, L. J.; Gudiksen, M. S.; Wang, J.; Lieber, C. M. Diameter-controlled synthesis of single-crystal silicon nanowires. Appl. Phys. Lett. 2001, 78 (15), 2214-2216.

17. Krogstrup, P.; Curiotto, S.; Johnson, E.; Aagesen, M.; Nygård, J.; Chatain, D. Impact of the liquid phase shape on the structure of III-V nanowires. Phys. Rev. Lett. 2011, 106 (12), 125505.

18. Nolan, M.; O'Callaghan, S.; Fagas, G.; Greer, J. C.; Frauenheim, T. Silicon nanowire band gap modification. Nano Lett. 2007, 7 (1), 34-38.

19. Zheng, Y.; Rivas, C.; Lake, R.; Alam, K.; Boykin, T. B.; Klimeck, G. Electronic properties of silicon nanowires. IEEE Trans. Electron Devices 2005, 52 (6), 1097-1103.

20. Guichard, A. R.; Barsic, D. N.; Sharma, S.; Kamins, T. I.; Brongersma, M. L. Tunable light emission from quantum-confined excitons in TiSi2-catalyzed silicon nanowires. Nano Lett. 2006, 6 (9), 2140-2144.

21. Ziad, Y. A.; Zhiming, M. W.; Jihoon, H. L.; Gregory, J. S. Observation of Ga droplet formation on (311)A and (511)A GaAs surfaces. Nanotechnology 2006, 17 (16), 4037.

22. Miroslav, K.; Tomáš, Č.; Eva, B.; Jan, Č.; Tomáš, Š. Self-limiting cyclic growth of gallium droplets on Si(111). Nanotechnology 2008, 19 (47), 475606. 
23. Yang, H.; LaBella, V. P.; Bullock, D. W.; Ding, Z.; Smathers, J. B.; Thibado, P. M. Activation energy for Ga diffusion on the GaAs(001)-(2×4) surface: an MBE-STM study. J. Cryst. Growth 1999, 201-202, 88-92.

24. Mo, Y. W.; Kleiner, J.; Webb, M. B.; Lagally, M. G. Activation energy for surface diffusion of Si on Si(001): A scanning-tunneling-microscopy study. Phys. Rev. Lett. 1991, 66 (15), 1998-2001.

25. Curiotto, S.; Leroy, F.; Cheynis, F.; Müller, P. Surface-dependent scenarios for dissolutiondriven motion of growing droplets. Sci. Rep. 2017, 7 (1), 902.

26. Nemcsics, Á.; Heyn, C.; Stemmann, A.; Schramm, A.; Welsch, H.; Hansen, W. The RHEED tracking of the droplet epitaxial grown quantum dot and ring structures. Mater. Sci. Eng. B 2009, 165 (1-2), 118-121.

27. Harmand, J.-C.; Patriarche, G.; Glas, F.; Panciera, F.; Florea, I.; Maurice, J.-L.; Travers, L.; Ollivier, Y. Atomic Step Flow on a Nanofacet. Phys. Rev. Lett. 2018, 121 (16), 166101.

28. Chou, Y.-C.; Hillerich, K.; Tersoff, J.; Reuter, M.; Dick, K.; Ross, F. Atomic-scale variability and control of III-V nanowire growth kinetics. Science 2014, 343 (6168), 281-284.

29. Jacobsson, D.; Panciera, F.; Tersoff, J.; Reuter, M. C.; Lehmann, S.; Hofmann, S.; Dick, K. A.; Ross, F. M. Interface dynamics and crystal phase switching in GaAs nanowires. Nature 2016, 531 (7594), 317.

30. Stowell, M.; Hutchinson, T. Nucleation kinetics in thin film growth: II. Analytical evaluation of nucleation and growth behaviour. Thin Solid Films 1971, 8 (1), 41-53.

31. Venables, J. A. Nucleation calculations in a pair-binding model. Phys. Rev. B 1987, 36 (8), $4153-$ 4162.

32. Canniff, J. C.; Jeon, S.; Huang, S.; Goldman, R. S. Formation and coarsening of near-surface Ga nanoparticles on SiNx. Appl. Phys. Lett. 2015, 106 (24), 243102.

33. Lewis, B.; Halpern, V. Surface diffusion capture in nucleation theory. J. Cryst. Growth 1976, 33 (1), 39-52.

34. Kley, A.; Ruggerone, P.; Scheffler, M. Novel Diffusion Mechanism on the GaAs(001) Surface: The Role of Adatom-Dimer Interaction. Phys. Rev. Lett. 1997, 79 (26), 5278-5281.

35. Nao-haru, S.; Toshiro, I.; Yoshifumi, K. Desorption of the Excess Gallium Atoms at the Surface of Gallium Arsenide and Application to Atomic Layer Epitaxy. Jpn J. Appl. Phys. 1989, 28 (2A), L287.

36. Oehler, F.; Cattoni, A.; Scaccabarozzi, A.; Patriarche, G.; Glas, F.; Harmand, J.-C. Measuring and modeling the growth dynamics of self-catalyzed GaP nanowire arrays. Nano Lett. 2018, 18 (2), 701 708.

37. Vukajlovic-Plestina, J.; Kim, W.; Ghisalberti, L.; Varnavides, G.; Tütüncuoglu, G.; Potts, H.; Friedl, M.; Güniat, L.; Carter, W.; Dubrovskii, V. Fundamental aspects to localize self-catalyzed III-V nanowires on silicon. Nat. Commun. 2019, 10 (1), 869.

38. Venables, J. A.; Spiller, G. D. T.; Hanbucken, M. Nucleation and growth of thin films. Rep. Prog. Phys. 1984, 47 (4), 399.

39. Heyn, C.; Stemmann, A.; Schramm, A.; Welsch, H.; Hansen, W.; Nemcsics, Á. Regimes of GaAs quantum dot self-assembly by droplet epitaxy. Phys. Rev. B 2007, 76 (7), 075317.

40. Balducci, G.; Gigli, G.; Meloni, G. Dissociation energies of the Ga2, In2, and Galn molecules. J. Chem. Phys. 1998, 109 (11), 4384-4388.

41. Leon, R.; Senden, T. J.; Kim, Y.; Jagadish, C.; Clark, A. Nucleation Transitions for InGaAs Islands on Vicinal (100) GaAs. Phys. Rev. Lett. 1997, 78 (26), 4942-4945.

42. Zheng, H.; Smith, R. K.; Jun, Y.-W.; Kisielowski, C.; Dahmen, U.; Alivisatos, A. P. Observation of single colloidal platinum nanocrystal growth trajectories. Science 2009, 324 (5932), 1309-1312.

43. Losurdo, M.; Suvorova, A.; Rubanov, S.; Hingerl, K.; Brown, A. S. Thermally stable coexistence of liquid and solid phases in gallium nanoparticles. Nat. Mater. 2016, 15 (9), 995.

44. Oliphant, T. E. Python for scientific computing. Comput. Sci. Eng. 2007, 9 (3), 10-20.

45. Walt, S. v. d.; Colbert, S. C.; Varoquaux, G. The NumPy array: a structure for efficient numerical computation. Comput. Sci. Eng. 2011, 13 (2), 22-30. 
46. Millman, K. J.; Aivazis, M. Python for scientists and engineers. Comput. Sci. Eng. 2011, 13 (2), $9-12$.

47. Bradski, G.; Kaehler, A. Learning OpenCV: Computer vision with the OpenCV library. " O'Reilly Media, Inc.": 2008.

48. Van der Walt, S.; Schönberger, J. L.; Nunez-Iglesias, J.; Boulogne, F.; Warner, J. D.; Yager, N.; Gouillart, E.; Yu, T. scikit-image: image processing in Python. PeerJ 2014, 2, e453.

49. Behnel, S.; Bradshaw, R.; Citro, C.; Dalcin, L.; Seljebotn, D. S.; Smith, K. Cython: The best of both worlds. Comput. Sci. Eng. 2011, 13 (2), 31-39.

50. Hunter, J. D. Matplotlib: A 2D graphics environment. Comput. Sci. Eng. 2007, 9 (3), 90-95.

51. Serra, J. Morphological filtering: an overview. Signal Process. 1994, 38 (1), 3-11.

52. Kimori, Y.; Baba, N.; Morone, N. Extended morphological processing: a practical method for automatic spot detection of biological markers from microscopic images. BMC Bioinf. 2010, 11 (1), 373.

53. Kapur, J. N.; Sahoo, P. K.; Wong, A. K. A new method for gray-level picture thresholding using the entropy of the histogram. Comput. Vis. Graph. Image Process. 1985, 29 (3), 273-285. 